PART IV

SCIENTIFIC DEDICATION OF THE 107-INCH REFLECTOR 


\section{REMARKS MADE AT THE SCIENTIFIC DEDICATION OF THE 107-INCH REFLECTOR, OCTOBER 30, 1969}

Dr. Harlan Smith: Not many large telescopes in the world have been dedicated twice. Yet this may not be such a bad thing to do. The convention has long existed of christening a baby at birth and baptizing it later; in this sense the 107-inch telescope was christened almost a year ago, and today might be considered its baptism. Indeed, the parallel is rather apt, because christening is a naming - a formal recognition of coming into existence - whereas a baptism in the traditional sense of the word is a dedication to a certain path of life.

Our first dedication was a christening, a coming-into-existence affair. Those who have been closely involved in the construction of a large telescope can appreciate the number of problems that arise, the moments when there is even doubt whether the job will be finished. So the first completion in a real sense was the birth, the proper christening time of the instrument. At the November, 1968, dedication the telescope was essentially complete, but several problems delayed the start of observing until March 7, 1969, after which time it was appropriate to consider a scientific baptism - its dedication to research.

A more practical rationale for our second dedication was the large number of people who were in some way connected with this telescope. There was scarcely room for so many here; to have a substantial scientific delegation in addition would have overcrowded the facilities and changed the character of the first program. On the other hand, this second dedication reflects the facts that we have present a quorum of the world's planetary astronomers, and are baptizing the world's first very large telescope conceived and dedicated in considerable measure for planetary research.

It is also appropriate that we should be here dedicating this telescope in part for planetary research because McDonald Observatory was, during the lean years of planetary work, one of the few strongholds of continued effort along such lines. Beginning about the time Professor Kuiper came to Chicago and continuously to the present, the McDonald Observatory has devoted an appreciable fraction of its 82-inch time to solar system problems, a statement which could probably not be made of any other large telescope in the world. But the large group of astronomers present at this meeting gives heartening evidence for the rebirth of interest in planetary subjects, this time with a strongly astrophysical flavor which usually requires large telescopes to gather enough light for the necessary types of observation. Such studies at McDonald using the 82-inch Struve reflector (and the 36-inch reflector to a lesser degree) helped greatly to put on a firm foundation the basic astrophysics of the planets and satellites of the solar system: colors, spectra, atmospheres, and surface properties of the moon, planets, satellites, asteroids, and comets - even zodiacal light and gegenschein.

About five years ago we came to feel that the increasing amount of planetary work needing to be done was more than could be handled by the 82 - and 36 -inch reflectors, along with increasing needs also in stellar, interstellar, and extragalactic astronomy. The proper solution to the shortage of planetary observing time was of course to 
augment the world's supply of large telescopes. The National Aeronautics and Space Administration concurred; two men who especially contributed during this early stage at NASA were Dr. Urner Liddell and Dr. Ronald Schorn.

Thus, late in 1964, it was agreed between the University of Texas and NASA that an additional large telescope should be erected here at the McDonald Observatory, which would normally devote at least a quarter of its time to solar system - mainly planetary - research. A year and a half were needed to work out arrangements and detailed design; three and a half years ago fabrication began on the telescope and the dome. That work took only two and a half years, and the telescope began to be installed in mid-1968. Beginning March 7, 1969, the drives were effectively turned on, and spectra of Mars showing water vapor were obtained on the first several nights. Since then the 107-inch has been in almost continuous use, principally at the coude focus where the planetary instruments are concentrated.

The first of these coude instruments is the giant horizontal coude spectrograph which fills most of the floor directly beneath us. In the coude slit room is the Connestype interferometic spectrometer of Dr. Reinhard Beer (JLP), currently working from 3 to $5 \mu$ with extremely high resolution. Medium-dispersion but therefore faster interferometric spectrometers are also used in the slit room (Dr. Rudi Hanel of Goddard and Dr. Andrew Potter of Manned Spacecraft Center).

This past summer we added a new coude focus area at the south end of the observing floor, to provide space for the principal equipment for laser ranging on the lunar target left by the Apollo 11 astronauts in July. The laser beam sent out almost daily from the 107-inch reflector has a divergence typically of only several seconds of arc; the retroreflector on the moon returns about $10^{-8}$ to $10^{-9}$ of this light toward the earth, diverging in a wider cone of which again a comparably small fraction is intercepted by the telescope. The few remaining photons are recorded in the laser room by a photocell and their time of travel measured to about a nanosecond. This system has been in operation since August, initially under the supervision of Dr. Brian Warner.

In addition to such solar-system coude programs, the telescope obviously has many other potential uses. In particular at the four Cassegrain focal positions (three of them reached by rotating the first coude flat mirror inside the tube) we propose to do stellar and extragalactic work. The $f / 18$ Cassegrain has been brought into initial operation over the last few weeks; the $f / 9$ will follow shortly. To refer to only one example of our Cassegrain plans, those of you who on the way to this session visited the University of Texas Radio Astronomy Observatory under the direction of Dr. James Douglas, saw an installation which among other activities will soon begin to produce thousands of radio source positions accurate to about a second of arc. A major use of the 107-inch telescope during dark of the moon will be the optical study of these radio sources, including Seyfert galaxies, quasars, and similar objects. Over the next few decades this telescope is thus not likely to be idle.

But I want to emphasize again that more than half of the support necessary to build the 107-inch reflector was provided by NASA (principally the Lunar and Planetary Office); the University of Texas provided more than half of the remainder, and the National Science Foundation the rest. This support over the last five years has been 
deeply appreciated; and has made possible the telescope and associated new McDonald facilities. Now, with its successful coming into operation, there exists the first large telescope planned with one of its major roles in support of planetary research. As long as there are such programs which can usefully be done with a large ground-based telescope, the 107-inch will be deeply involved.

It is especially appropriate at this time to introduce the man who over the last three years has had the most to do with supporting the telescope administratively and financially, Dr. William Brunk of the National Aeronautics and Space Administration, who through his direction of the planetary research support programs of NASA has been our chief reliance in this area.

Dr. Brunk: After that introduction, I must admit that there were several times when I wondered whether I would actually stand under this telescope. It's very nice to see it here, and better still to know that we're already beginning to get some important and interesting information from it. A further bonus is the fact that we have been able on such short notice to come in with an entirely new program such as the lunar laser and have it operating so soon in a relatively smooth manner.

I do want to emphasize that we at NASA have realized more and more with the passage of time that to really gain a thorough understanding of our solar system, the moon and planets, it is necessary to rely not only on spacecraft which can give unique answers but also on observations made from the earth and from the vicinity of the earth (from the earth's atmosphere, or rockets or satellites immediately above the atmosphere). For this reason we have put a great deal of effort into trying to foster the maximum ground-based program in planetary astronomy, and one of the fruits of the program has been this telescope. It happens to be the largest we have been able to undertake; it is not the only one, as the astronomers here are well aware. Another major NASA-supported instrument is approaching completion near the top of Mauna Kea on the island of Hawaii - an 88-inch telescope which looks quite different from this one, and stands at an altitude of nearly $14000 \mathrm{ft}$. Also we were able a few years ago to build for Dr. Kuiper a very fine instrument for doing primarily photography and photometry of the planets. In addition to this we have been fortunate enough to support construction of a few minor telescopes, as mentioned in the discussion on planetary patrols this morning, comprising about six stations scattered around the world. And we look forward to being able to help astronomers who are interested in a few other areas that have not been worked on enough in the past. One of these areas is the far infrared, and we accordingly hope to be able to support some major instrumentation for infrared astronomy. We are also very interested in making maximum use of radio and radar astronomy, which have certainly shown great benefit for planetary programs.

Concerning these major instruments in which the major portion of the NASA investment has been primarily for planetary research (even though of course they are also useful for stellar and other work), we are anxious to have these instruments available to guest observers when possible. And that has already been true here. The 
lunar laser experiment is really a guest-investigator experiment. Reinhard Beer with his large interferometer is from the Jet Propulsion Laboratory. Likewise Ron Schorn from JPL comes to McDonald to observe planets, mainly with the 82 -inch reflector to be sure, and people from NASA-Goddard have also been using these instruments. Indeed, as a matter of principle we hope in all cases where we are able to help an institution with an instrument, that it becomes accessible to people with good, scientifically sound programs in planetary astronomy, regardless of the institution from which they come.

Dr. Harlan Smith: Thank you, Dr. Brunk; we agree with these sentiments.

This telescope is new in several ways. In particular it was designed to be integrated with a computer from the beginning, both for control and data-taking functions. To operate the ever-increasingly sophisticated equipment, both of the telescope and its auxiliary instruments, needs a larger and more sophisticated staff than was formerly the case. The staff burden is further increased because we now schedule both large telescopes nearly 24 hours a day, so much work being done in the infrared where the difference between day and night is almost immaterial. Finally, as all of you know, the Observatory works every day of the year, which further adds to the burden. It would be quite impossible to run a facility like this with our relatively small staff on such a schedule, if it were not for the dedication of a number of people, of whom I should mention particularly the Superintendant, Curtis Laughlin, and his three capable assistants, David Dittmar, George Grubb, and Dr. Maurice Marin; also Dr. Edwin Barker, Resident Supervisor of the planetary programs here. I want to take this rare opportunity to thank them for their extraordinarily devoted work, and to make a passing comment on it. A federal wage-hour law forbids people engaged in interstate commerce - and apparently our photons come from out-of-state - to be worked more than 40 hours a week. Fortunately for the sake of the observatory, this rule does not apply to supervisors!

I commented earlier that McDonald has had a long history of planetary work. One man, above all others together, made that possible - in fact, did most of the work himself either directly or through his immediately co-working graduate students. I refer of course to Professor Gerard Kuiper, now of the University of Arizona, Director of the Lunar and Planetary Laboratory. It is totally appropriate for this dedication that Professor Kuiper should be here to address us in a baptismal sense about the role of ground-based astronomy.

Dr. Kuiper: Mrs. Kuiper and I are happy to be back - almost home. We spent a lot of time here from about 1938 until about 1960, and even after moving to the University of Arizona have had the privilege of using the 82-inch from time to time.

A few comments might be especially appropriate on this occasion.

With the pressures of the large national space programs, a redoubled effort of course is needed in ground-based planetary astronomy. During the past dozen years or so the space programs, from the point of view of a scientist interested in planets, 
have matured in a very interesting way, in that a balanced work load can now be assigned to the six successively more complex modes of planetary research: groundbased, aircraft-based, balloon-based, rocket-based, satellite-based, and deep space probes. Each of these six technologies can make its own special contribution. Each simpler one can and should be used to the maximum effectiveness in its workload, because of the comparative economy, smaller required manpower, and quicker results frequently obtainable with the less complex techniques.

So, to a scientist who has watched the development of planetary studies for the past twenty years or more, I think we can truly say that the present age is a very fortunate one in which all six approaches are open to the scientist where necessary, and the individual interest can best be served, and the maximum contribution made.

The 107-inch here outclasses technically its 82 -inch neighbor on the mountain. But the 82-inch Struve reflector has its own history, and perhaps I may be permitted to comment about some of the planetary programs that have been carried out with it (not to mention the tremendous output of stellar spectroscopy and programs of galactic structure carried out by Struve and dozens of collaborators since the inauguration of the 82-inch just thirty years ago).

Let me then reflect briefly on some of the contributions of this older and smaller sister here on the mountain. In the first place, an atmosphere of methane was discovered with it on the satellite Titan in 1944, the only satellite for which a gaseous atmosphere has been shown to exist. Carbon dioxide was identified on Mars with the 82-inch in 1947; the polar caps of Mars were found to show the reflection spectrum of very fine, very cold snow with it around 1950. The fifth satellite of Uranus and the second satellite of Neptune were discovered with it in 1947-48. The reflection spectrum of the dark areas of Mars was found to be not that of vegetation containing water, nor in fact were the dark areas of Mars found to be green. This was done in the late 40's and early 50's. The 8270 band of the pressure-induced dipole spectrum of hydrogen was discovered on Uranus and Neptune in the early 50's. The fifth satellite of Jupiter was photographed for the first time with the 82 -inch; it had been seen but not photographed; from this we could get accurate astrometric positions. An extensive program of lunar studies was begun with the 82-inch in 1953 and carried through 1954, which led to a major 1954 publication in the Proceedings of the National Academy of Sciences, in which lunar timescales were developed. It put the age of the maria at 4.5 billion years, and indicated that the maria may not have been entirely caused by impacts but rather by magmas released as a result of major impacts; these impacts may not have come from meteorites or asteroids or comets, but from a satellite ring around the earth. Finally, a program of lunar photography was begun in the middle 50 's that led to a series of lunar atlases; systems of coordinates were combined with these photographs. This led to the production of the orthographic atlas of the moon, which has been the basis of modern front-side lunar cartography. As a personal interjection, when I was recently in Houston with the Apollo 11 astronauts, I was naturally pleased that the only atlas in their office was the orthographic atlas of the moon. (It was an unannounced visit!)

I am sure that the opportunities of this mighty 107-inch will be larger than they were 
for the 82 -inch. Already the instrument has begun to show its power in several major programs to which Dr. Smith has made reference.

Now since these proceedings are in a sense part of the 40th IAU Symposium, I might make just one brief remark about some thoughts that occurred to me during this Symposium, that have a definite bearing on the purposes of a telescope such as this.

If the 40th IAU Symposium may have left the impression with some of you that even incorrect observations, such as the microwave phase-curves of Venus, can lead to correct answers by good theoreticians, then I think I should point out a counterpart conclusion that greatly outweighs that impression. Specifically, much of planetary astronomy in the last decade has been the clearing away of incorrect ghosts left by earlier publications. Fortunately this audience is largely composed of planetary scientists, so this is really criticism in the family, but nevertheless let me mention a few of the skeletons in our planetary closet that we had to remove: the rotation of Mercury; the surface temperature and the rotation of Venus; the pressure and the rotational temperature of the Venus atmosphere; the canals and the surface vegetation on Mars; the spectroscopic rotation of Jupiter; the degree of penetration of the infrared radiation into the Jupiter atmosphere; dust and ice on the moon; the acceleration of Phobos around Mars. Other examples could be cited of hasty conclusions based on inadequate data. I think this strengthens the central convictions which must have led to the erecting of this mighty telescope; namely that, in the first place, planetary astronomy is an empirical science of great complexity rather like geophysics in its many branches. Thus with the large national investments now being made in the space programs, it behooves planetary astronomers to make sure that the data they produce are relevant to the great scientific problems and that they are reliable. The 61-inch telescope of the Lunar and Planetary Laboratory was the first telescope sponsored by NASA; the McDonald 107-inch and the Hawaii 88-inch followed. The completion of this mighty 107-inch on this high and dry mountain is a great event for planetary science.

I congratulate NASA and the University of Texas that their wise decisions have led to construction of this instrument, and look forward to great results from the various programs to which it is dedicated. 